\title{
Light transport in cold atoms: the fate of coherent backscattering in the weak localization regime
}

\author{
D. Wilkowski ${ }^{1}$ \\ Y. Bidel, T. Chanelière, R. Kaiser, B. Klappauf, G. Labeyrie, \\ C.A. Müller and Ch. Miniatura \\ Laboratoire Ondes et Désordre, FRE 2302 du CNRS, 1361 route des Lucioles, \\ Valbonne F-06560, France
}

\begin{abstract}
The recent observation of coherent backscattering (CBS) of light by atoms has emphasized the key role of the velocity spread and of the quantum internal structure of the atoms. Firstly, using highly resonant scatterers imposes very low temperatures of the disordered medium in order to keep the full contrast of the CBS interference. This criterion is usually achieved with standard laser cooling techniques. Secondly, a non trivial internal atomic structure leads to a dramatic decrease of the CBS contrast. Experiments with Rubidium atoms (with a non trivial internal structure) and with Strontium (with the simplest possible internal structure) show this behaviour and confirm theoretical calculations.
\end{abstract}

Key words: Multiple scattering, Atom, Cooling

After a few scattering mean free path $\ell$, a wave (with wavenumber $k=2 \pi / \lambda$ ) propagating in an opaque medium rapidly looses the memory of its initial direction. At this scale, intensity propagation is often described as a diffusion process. However this description discards an important phenomenon: interference between multiply scattered waves. It is now known that interference alters the wave transport and can, under suitable conditions, bring it to a complete stop. This is the Anderson (or strong) localization regime, where the diffusion is suppressed [1]. For twenty years, there have been tremendous experimental as well as theoretical efforts to study interference effects in the multiple scattering regime [2].

A hallmark in this field is the CBS cone which is observed as a reflection

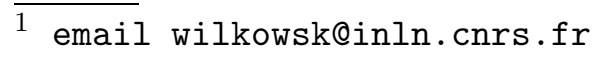


peak with angular width $\simeq 1 / k \ell$ at backscattering. It corresponds to the incoherent sum of two-waves interference between a possible multiple scattering path amplitude and its reversed counterpart [3]. This interferential increase of the configuration-averaged diffuse reflection off a disordered sample depends on the nature of the scatterer but also, for vectorial waves like light, on the input/output polarization channel. For classical spherically-symmetric scatterers, the maximum enhancement factor of the CBS cone is 2 in the $h \| h$ helicitypreserving polarization channel, independently of the geometrical shape of the scattering medium [4]. This is so for two reasons. First, in the $h \| h$ channel single scattering is suppressed : for spherically-symmetric scatterers, the polarization is conserved at backscattering, like in specular reflection off a mirror. Second, in the absence of a magnetic field, the amplitudes of the interfering paths are exactly equal at backscattering by virtue of the reciprocity theorem $[5]$.

We will see that the observation of a CBS cone with a maximum contrast on a resonant atomic gas, however imposes severe restrictions on the external as well as internal degrees of freedom of the atoms. In section 1 we review some effects related to moving resonant scatterers. In section 2 we show how the internal structure of the atomic scatterer reduces the CBS contrast. In section 3 experimental results, obtained in the weak localization regime $k \ell \gg 1$, are presented. They confirm theoretical predictions about the role of the internal structure.

\section{CBS with resonant scatterers}

\subsection{Dynamical breakdown of CBS enhancement factor: Double scattering model}

When the scattering medium is made of moving particles, and this is the case in an atomic cloud, the amplitudes of the direct and reverse scattering sequences are no more linked by reciprocity and one observes a dynamical breakdown of the CBS effect [6]. This reduction of the CBS enhancement factor depends on the velocity distribution of the particles and we can assert very generally that the interference contrast will not be much affected provided the velocity spread will not be too large. For moving resonant scatterers, we will see that "not too large" imposes a stringent condition which will require laser cooling techniques to be fulfilled.

As a simplistic starting model, we neglect light polarization effects (scalar wave approximation) and describe atoms as highly resonant isotropic scatterers. The atomic resonance is characterized by a transition frequency $\omega_{0}$ in the optical domain and a transition width $\Gamma$ (typically $\frac{\omega_{0}}{\Gamma} \simeq 10^{8}$ ). The scatterers are then 


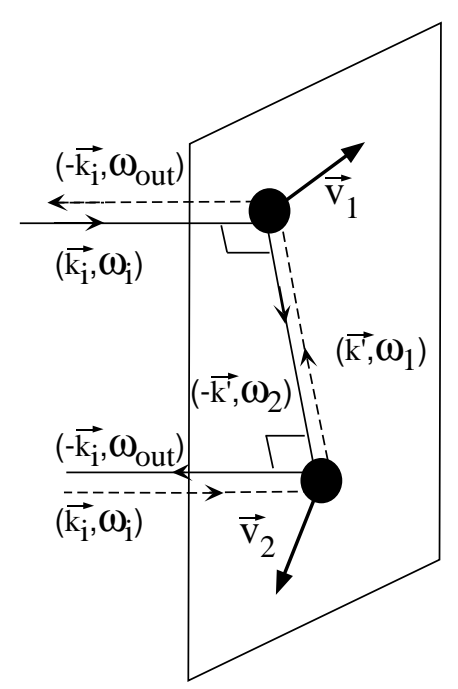

Fig. 1. Double scattering process with moving atoms. The atoms move in an orthogonal plane respect to the incident wave-vector. We have $\omega_{1}=\omega-\left(\mathbf{k}_{\mathbf{i}}-\mathbf{k}^{\prime}\right) \mathbf{v}_{\mathbf{1}}$, $\omega_{2}=\omega-\left(\mathbf{k}_{\mathbf{i}}+\mathbf{k}^{\prime}\right) \mathbf{v}_{\mathbf{2}}$ and $\omega_{\text {out }}=\omega_{1}-\left(\mathbf{k}_{\mathbf{i}}+\mathbf{k}^{\prime}\right) \mathbf{v}_{\mathbf{2}} \cdot \mathbf{k}_{\mathbf{i}}\left(-\mathbf{k}^{\prime}\right)$ is the incident (intermediate) wave-vector.

fully characterized by their complex electrical polarizability $\alpha(\omega)$, with:

$$
\alpha(\omega)=-\frac{3 \pi \Gamma c^{3}}{\omega_{0}^{3}}\left(\frac{1}{\omega-\omega_{0}+i \frac{\Gamma}{2}}\right)
$$

since the scattering differential cross-section is $\frac{d \sigma}{d \Omega}=\frac{\sigma}{4 \pi}$ where the total crosssection is $\sigma=\frac{k^{4}|\alpha|^{2}}{6 \pi}$. The velocity distribution of atoms has too major consequences: first the atomic response at each scattering has to be evaluated at a frequency $\omega-\mathbf{k v}$ which is randomly Doppler-shifted. This means that each scattering has a variable strength and that the wave experiences a random phase-kick. Furthermore, after scattering is completed, the wave has a frequency $\omega^{\prime}=\omega-\left(\mathbf{k}-\mathbf{k}^{\prime}\right) \mathbf{v}$. Scattering is inelastic and propagation in an effective medium (until the following scattering) occurs with an optical index evaluated at random frequency. As an overall conclusion, there will be a random strength imbalance and a random dephasing between direct and reverse scattering sequences, leading to a reduction of the CBS interference. Let us illustrate this dynamical breakdown for a peculiar double scattering path (see figure 1). We consider an incoming resonant light wave (frequency $\omega=\omega_{0}$, wave-vector $\mathbf{k}_{\mathbf{i}}$ ) and, to simplify the discussion, we only consider the effect of dephasing between the paths. The total phase shift at backscattering $\left(\mathbf{k}_{\text {out }}=-\mathbf{k}_{\mathbf{i}}\right)$ is

$$
\Delta \phi=\Delta \phi_{\text {pro }}+\Delta \phi_{\text {sca }}
$$

Where $\Delta \phi_{\text {pro }}$ corresponds to the dephasing accumulated during propagation in the effective medium and $\Delta \phi_{\text {sca }}$ the dephasing accumulated at scattering events. In a dilute medium $(n|\alpha| \ll 1$, where $n$ is the spatial density), the 
expressions of $\Delta \phi_{\text {pro }}$ et $\Delta \phi_{s c a}$ are quite straightforward:

$$
\Delta \phi_{\text {pro }}=\frac{n_{r}\left(\omega_{1}\right) \omega_{1}-n_{r}\left(\omega_{2}\right) \omega_{2}}{c} L
$$

and

$$
\Delta \phi_{s c a}=f\left(\omega-\mathbf{k}_{\mathbf{i}} \mathbf{v}_{\mathbf{1}}\right)+f\left(\omega_{1}-\mathbf{k}^{\prime} \mathbf{v}_{\mathbf{2}}\right)-f\left(\omega-\mathbf{k}_{\mathbf{i}} \mathbf{v}_{\mathbf{2}}\right)-f\left(\omega_{2}+\mathbf{k}^{\prime} \mathbf{v}_{\mathbf{1}}\right)
$$

with

and

$$
f(\omega)=\operatorname{Arg}(\alpha)=\arctan \left(\frac{\Gamma}{2\left(\omega_{0}-\omega\right)}\right)
$$

$$
\begin{aligned}
& \omega_{1}=\omega-\left(\mathbf{k}_{\mathbf{i}}-\mathbf{k}^{\prime}\right) \mathbf{v}_{\mathbf{1}} \\
& \omega_{2}=\omega-\left(\mathbf{k}_{\mathbf{i}}+\mathbf{k}^{\prime}\right) \mathbf{v}_{\mathbf{2}}
\end{aligned}
$$

The distance $L$ between scatterers is of the order of the scattering mean free path $\ell\left(\omega_{0}\right)=\frac{1}{n \sigma\left(\omega_{0}\right)}$. The optical index $n_{r}(\omega)$ is given by

$$
n_{r}(\omega) \simeq 1+n \frac{\operatorname{Re}(\alpha(\omega))}{2}
$$

At first order in velocities, we get:

$$
\Delta \phi_{\text {pro }} \simeq-\frac{1}{\Gamma}\left(\left(\mathbf{k}^{\prime}-\mathbf{k}_{\mathbf{i}}\right) \mathbf{v}_{\mathbf{1}}+\left(\mathbf{k}^{\prime}+\mathbf{k}_{\mathbf{i}}\right) \mathbf{v}_{\mathbf{2}}\right)
$$

and

$$
\Delta \phi_{s c a} \simeq \frac{4}{\Gamma} \mathbf{k}_{\mathbf{i}}\left(\mathbf{v}_{\mathbf{1}}-\mathbf{v}_{\mathbf{2}}\right)
$$

Averaging over the Gaussian velocity distribution of the two atoms leads to the interference contrast:

$$
C\left(\left\langle v^{2}\right\rangle\right)=1+\langle\cos (\Delta \phi)\rangle_{\mathbf{v}_{\mathbf{1}}, \mathbf{v} \mathbf{2}}=1+\exp \left(-6 \frac{k^{2}\left\langle v^{2}\right\rangle}{\Gamma^{2}}\right)
$$

where

$$
k \simeq\left|\mathbf{k}_{\mathbf{i}}\right| \simeq\left|\mathbf{k}^{\prime}\right|
$$

Expression 3 shows that, to preserve the double scattering CBS effect, one needs :

$$
\sqrt{\left\langle v^{2}\right\rangle} \ll \Gamma / k
$$

For scattering sequences of higher orders, one can argue that the scattering phaseshift roughly performs a random walk of step of order of $\frac{k \sqrt{\left\langle v^{2}\right\rangle}}{\Gamma}$, thus increasing as $\sqrt{N}$ with scattering order $N$. This puts a more stringent condition 


\section{Table 1}

\begin{tabular}{|c|c|c|c|c|}
\hline Atom & $\lambda(n m)$ & $\frac{\Gamma}{2 \pi}(M H z)$ & $k \sqrt{\left\langle v^{2}\right\rangle}\left(\Gamma^{-1}\right)$ & $C\left(\left\langle v^{2}\right\rangle\right)$ \\
\hline $\mathrm{Rb}$ & 780 & 6 & 0.04 & 2.0 \\
\hline $\mathrm{Sr}$ & 461 & 32 & 0.03 & 2.0 \\
\hline $\mathrm{Sr}$ & 689 & $7.10^{-3}$ & 1 & \\
\hline $\mathrm{He}^{*}$ & 1080 & 1.6 & 0.2 & 1.7 \\
\hline
\end{tabular}

Specific values of the double scattering contrast $C\left(\left\langle v^{2}\right\rangle\right)$ for different atoms and transitions. The cases of $\mathrm{Rb}, \mathrm{Sr}$ and $\mathrm{He}^{*}$ correspond to dipole-allowed transitions. For the intercombinaison line cooling of $\mathrm{Sr}$ (at $\lambda=689 \mathrm{~nm}$ ) the transition is too narrow and dynamical breakdown occurs.

on the required velocity spread to preserve the CBS effect at higher orders. For atoms, $\Gamma / k \simeq 10 \mathrm{~m} / \mathrm{s}$, which means that the atomic gas has first to be laser-cooled before observing CBS. This is done using a magneto-optical trap (MOT).

\subsection{Atoms cooling and trapping}

In a MOT, due to the combined action of the Zeeman and Doppler effects, a restoring force spatially traps the atoms at the zero of the magnetic field gradient and cools them in velocity space (for a review on cooling see e.g. W. Phillips [7]). The cooling action is based on a Doppler-induced differential radiation pressure force between contra-propagating laser beams. For a twolevel system in the low saturation limit and at low velocities $(k v \ll \Gamma)$, the average force is a pure friction force if the laser is red-detuned with respect to the transition $\left(\omega<\omega_{0}\right)$. At steady state, this cooling mechanism is balanced by the heating mechanism induced by the random character of photon absorption and emission processes. The equilibrium state is then characterized by a Gaussian velocity distribution with rms value:

$$
\sqrt{\left\langle v^{2}\right\rangle} \simeq \sqrt{\frac{\Gamma}{k} v_{r}}
$$

where the recoil velocity $v_{r}=\frac{\hbar k}{m}$ is the velocity change during a single absorption or emission event. For the usual dipole-allowed transitions, the recoil frequency shift is small with respect to the width of the transition

$$
k v_{r} \ll \Gamma
$$

Thus equation 4 is well fulfilled in a MOT and the dynamical breakdown

of the CBS effect should be negligible in most cases (see for example Table 
(a)
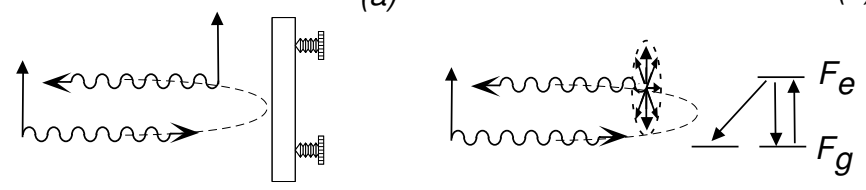

Fig. 2. For classical spherics scatterer, the backscattered wave conserves his polarization like in specular reflection off a mirror $(a)$. For a quantum scatterer the polarization is not anymore conserved, due to Raman transition (b).

1). Moreover, when $F_{g}>0$, extra cooling mechanics, the so-called Sisyphus cooling [8], lower the final rms velocity with respect to relation (5).

For $\mathrm{Rb}$, the MOT is loaded in a cell from a low-pressure thermal vapour and for Sr from an effusive atomic beam. The total number of trapped atoms is determined by a balance between trapping and loss mechanisms (e.g. atomatom collisions). It usually ranges from $10^{7}$ to $10^{10}$ atoms in standard operating conditions. However for localization experiments, it is crucial to achieve an optical depth at least larger than unity to reach the multiple scattering regime. This requirement is usually satisfied in a MOT using a resonant probe beam. It can be also interesting to achieve a high spatial density for strong localization experiments where $k \ell \simeq 1$ is demanded. Unfortunately, in most cases, multiple scattering is the dominant limiting factor [9] and spatial densities in a MOT are generally limited to a few of $10^{10}$ atoms $/ \mathrm{cm}^{3}$ implying $k \ell \gg 1$.

\section{Reduction of the CBS cone due to internal structure}

In section 1.1, we saw how the velocity distribution of atoms can alter the CBS contrast. Another important suppression mechanism of the CBS interference effects has been pointed out in reference [10]. It is related to the internal degrees freedom of the scatterers. In fact, the optical dipole transition connects two Zeeman-degenerate multiplets (in the absence of a magnetic field). The groundstate (resp. excited) multiplet has a total angular momentum $F_{g}$ (resp. $F_{e}$ ) and contains $2 F_{g}+1$ (resp. $\left.2 F_{e}+1\right)$ magnetic levels. To fully describe scattering, one needs to specify the initial and final groundstate levels. Two types of transitions can occur : those leaving the internal state unaffected (Rayleigh transitions) and those changing the internal state (Raman transitions). Thus, when calculating the CBS cone one has to take properly into account all those allowed mechanisms. Note that since all levels in a given multiplet have same energies, light scattering is always elastic. However scattering is no more described by the atomic polarizability alone and one now needs the full scattering tensor. In other words, non scalar features of scattering will become essential. It can be shown that the internal structure has two major consequences. First, because Raman transitions are unavoidable and since they are accompanied 

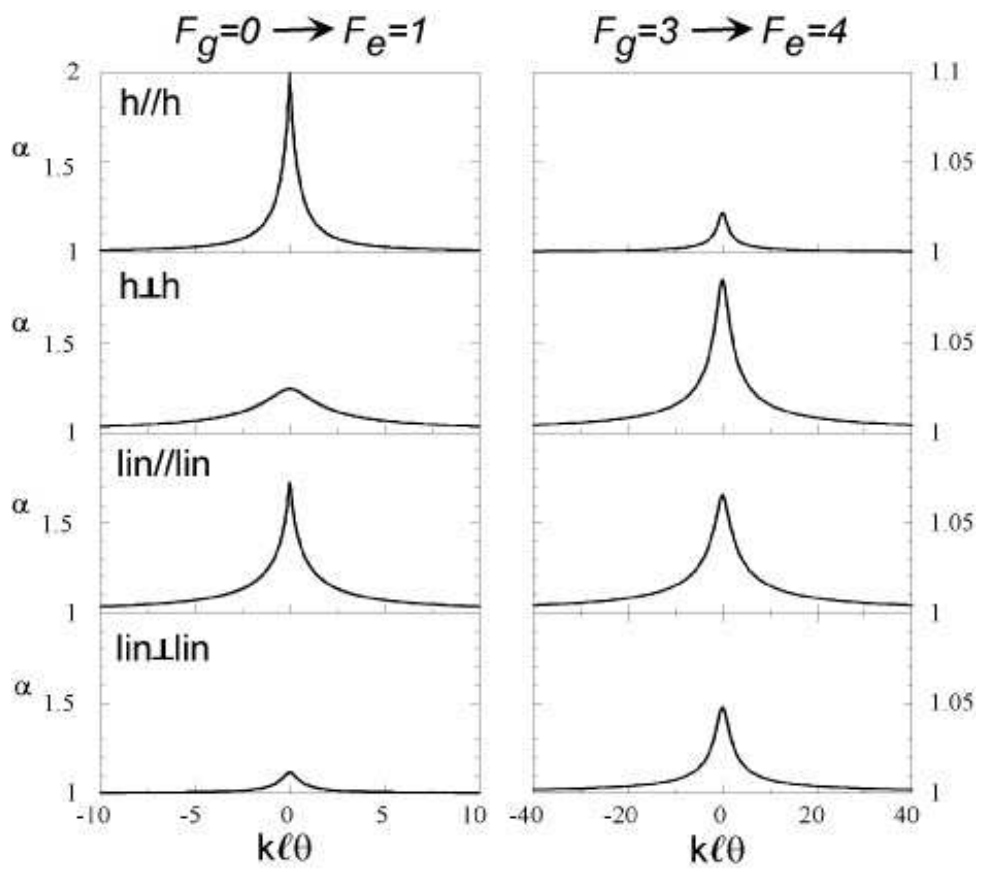

Fig. 3. CBS cones calculated in a semi-infinite homogeneous scattering medium. For the $F_{g}=3 \rightarrow F_{e}=4$ transition, the enhancement factor is systematical lower for all polarization channels when compared to the $F_{g}=0 \rightarrow F_{e}=1$ case where CBS cones are the same than for a classical dipole-like scatterer.

by a change of light polarization at backscattering (see Figure 2b), the single scattering contribution is no more suppressed in the $h \| h$ channel. This leads to a rather trivial CBS contrast reduction since the same would occur with classical non-spherically symmetric scatterers. Second, and this point is more subtle, the interfering amplitudes are no more linked in general by reciprocity. This is because under time-reversal the angular momentum of atoms has to be flipped. This leads to unbalanced amplitudes for the direct and reverse scattering sequences and to a decrease of the CBS contrast as soon as $F_{g}>0$ $[11,12]$. In the $h \| h$ channel, it can be shown that this contrast reduction can be attributed to the antisymmetric part of the scattering tensor [11]. For $F_{g}=0$ (absence of internal structure in the groundstate), one recovers the previous classical result. Figure 3 shows the CBS cone for the four usual polarization channels and for two different transitions in a semi-infinite medium. For the $F_{g}=0 \rightarrow F_{e}=1$ transition, cones shape and height are the same than for a classical dipole-like scatterer. For the $F_{g}=3 \rightarrow F_{e}=4$ transition, the enhancement factor is systematically lower for all polarization channels. 


\section{$3 \quad$ Experimental results}

The detailed experimental procedure for the CBS observation has been published elsewhere [13]. Briefly, the signal is obtained using a collimated resonant probe with a beam waist bigger than the size of the cloud $(\simeq 1 \mathrm{~cm})$. To avoid any effects linked to the saturation of the optical transition (non-linearities, inelastic radiation spectrum) [14], the probe intensity is weak (saturation parameter $s \ll 1)$. The scattered light is collected in the backward direction by placing a CCD camera in the focal plane of an achromatic doublet. The CBS cone plots shown in figure 4 are obtained in the $h \| h$ polarization. As predicted, the enhancement factor is strongly reduced for the $\mathrm{Rb}$ transition. However a quantitative comparison with theory calls for some care since the calculation assumes an homogeneous semi-infinite medium whereas the Rb experiment is made on a finite sample of Gaussian-distributed atoms. In this type of geometry, it is clear that the weight of long scattering paths are overestimated with respect to the finite size sample case. Besides, theoretical calculations show that the major contribution of the CBS cone comes from low scattering orders. Thus, considering only the single and double scattering events, the enhancement factor is predicted to be $\alpha_{t h}^{(2)}=1.17$ [10]. The experimental value thus interpolates nicely these two extreme predictions. For the $\mathrm{Sr}$ experiment, the enhancement factor is found to be $\alpha=1.86$, slightly lower the theoretical prediction $\alpha=2$. Several experimental issues can explain the difference; the finite angular resolution of the detection apparatus and the imperfect polarization channel isolation [15]. In figure 5, the experimental Sr CBS cone is compared to a Monte-Carlo simulation [15]. The agreement is clearly excellent.

\section{Conclusion}

To summarize, we have shown that the internal structure and the velocity distribution of resonant scatterers like atoms have a deep impact on the CBS effect. The dynamical breakdown induced by the motion of the scatterers can be made negligible at the expense of using laser-cooled atoms. Unfortunately, the internal structure irrevocably leads to very small enhancement factors in all polarization channels as soon as the atomic groundstate is degenerate. This has been evidenced by experiments on Rubidium. Restoration of a full interference contrast is obtained with non degenerate atoms like in the Strontium experiment. This should have interesting potentialities for wave localization experiments with cold atoms. For example, in the quest for Anderson localization (which could be obtained only at high density where $k l \approx 1$ ) where interferences play a crucial role, a $F_{g}=0 \rightarrow F_{e}=1$ transition appears to be a good choice. Is it now possible to increase the cloud density to reach the Anderson localization threshold? For this purpose, cooling strontium with the 

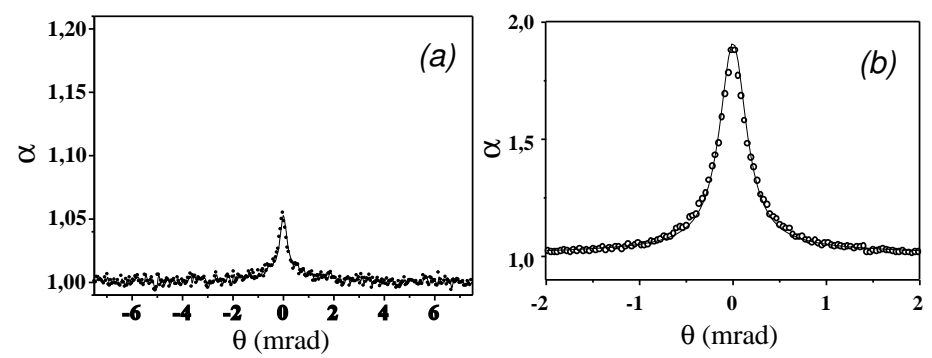

Fig. 4. Angular dependance of the CBS cone in the helicity preserving $h \| h$ polarization channel. Figure (a) corresponds to a $\mathrm{Rb}$ cloud of $4 \mathrm{~mm}$ of diameter and a optical depth of $b \simeq 30$ (the plain line connect two adjacent experimental points). The CBS cone on $\mathrm{Sr}$ is plotted on figure (b). For $\mathrm{Sr}$, the cold cloud has a diameter of $1 \mathrm{~mm}$ and an optical depth of $b \simeq 3$. The plain curve in figure (b) corresponds to a Monte-Carlo simulation done with the actual experimental parameters.

intercombination line in a dipole trap appears to be a promising technique $[16]$.

\section{Acknowledgments}

We gratefully acknowledge D. Delande for stimulating discussions and for Monte-carlo simulations on the Sr CBS cone. This work is funded by the CNRS and the PACA region.

\section{References}

[1] P.W. Anderson, Phys. Rev. 109, 1492 (1958). D.K. Ferry and S.M. Goodnick, Transport in Nanostructures, Cambridge University Press, New York (1997). S. Datta, Electronic Transport in Mesoscopic Systems, Cambridge University Press, Cambridge (1995).

[2] Mesoscopic Quantum Physics, ed. by E. Akkermans, G. Montambaux, J.-L. Pichard and J. Zinn-Justin, North-Holland, Amsterdam (1995). R. Berkovits and S. Feng, Phys. Rep. 238, 135 (1994). M.C.W. van Rossum and T.M. Nieuwenhuizen, Rev. Mod. Phys. 71, 313 (1999).

[3] Y. Barabanenkov, Izv. Vyssh. Uche. Zaved. Radiofiz. 16, 88 (1973). M. Van Albada and A. Lagendijk, Phys. Rev. Lett. 55, 2692 (1985). P. Wolf and G. Maret, Phys. Rev. Lett. 55, 2696 (1985). Y. Kuga and A. Ishimaru, J. Opt. Soc. Am. A 1, 831 (1985).

[4] D.S. Wiersma, M.P. van Albada, B.A. van Tiggelen and A. Lagendijk, Phys. Rev. Lett. 74, 4193 (1995). 
[5] B.A. van Tiggelen and R. Maynard, in Wave Propagation in Complex Media, IMA 96, edited by G. Papanicolaon (Springer, New-York), 252 (1997).

[6] A. Golubentsev, Zh. Eksp. Teor. Fiz. 86, 47 (1984).

[7] W. Philips, Rev. Mod. Phys. 70, 721 (1998).

[8] J. Dalibard and C. Cohen-Tannoudji, J. Opt. Soc. Am. B 6, 2023 (1989).

[9] T. Walker, D. Sesko and C. Wieman, Phys. Rev. Lett. 64, 408 (1990)

[10] T. Jonckheere, C.A. Müller, R. Kaiser, C. Miniatura and D. Delande, Phys. Rev. Lett. 85, 4269 (2000).

[11] C.A. Müller, T. Jonckeere, C. Miniatura and D. Delande, Phys. Rev. A 64, 053804 (2001).

[12] C.A. Müller and C. Miniatura, preprint: physics/0205029 , submitted to J. Phys. A (2002).

[13] G. Labeyrie, F. de Tomasi, J.C. Bernard, C.A. Müller, C. Miniatura and R. Kaiser , Phys. Rev. Lett. 83, 5266 (1999). G. Labeyrie, C.A. Müller, D. Wiersma, C. Miniatura and R. Kaiser, J. Opt. B : Quantum Semiclass. Opt. 2, $672(2000)$.

[14] V. Agranovich and V. Kravtsov, Phys. Rev. B 43 , 13691 (1991). A. Heiderich, R. Maynard and B. van Tiggelen, Opt. Comm. 115, 392 (1995).

[15] Y. Bidel, B. Klappauf, J.C. Bernard, D. Delande, G. Labeyrie, C. Miniatura, D. Wilkowski and R. Kaiser, Phys. Rev. Lett. 88, 203902 (2002).

[16] T. Ido, Y. Isoya and H. Katori , Phys. Rev. A 61, R033402 (2000). H. Katori, T. Ido and M. Gonokami, J. Phys. Soc. of Jap. 68, 2479 (1999). 